\title{
A periodic pricing model considering reference effect
}

\author{
Hui Yang* \& Chen Zhang \\ School of Economics and Management, Nanjing University of Science and Technology, Nanjing, Jiangsu, China
}

\begin{abstract}
The purpose of this paper is to investigate the optimal pricing strategies with reference effects in revenue management settings. We firstly propose a static pricing model with the properties of stochastic demand, finite horizon and fixed capacity, and prove the existence and uniqueness of the solution. Secondly, we extend the fixed pricing model to a periodic pricing model and incorporate a memory-based reference price in the demand function to investigate how the reference effect impacts on traditional revenue management decisions. We present numerical examples in both low demand situations and high demand situations for different levels of reference effects and different updating frequencies. The results show that the dynamic pricing strategies are superior to a static one even when reference effects are taken into consideration. We also provide some managerial insights including pricing directions, pricing dispersion and the optimal updating frequency for both demand situations.
\end{abstract}

Keywords: revenue management; pricing; reference effect; optimization

\section{INTRODUCTION}

Researches on dynamic pricing policies in revenue management situations have been pursued over two decades [1]. In the pioneering work, Gallego and van Ryzin (1994) and Bitran and Mondschein (1997) are both concerned with the pricing of a single product over a finite horizon and no replenishment opportunities [2][3]. They use different formulations: one is in a continuous-time setting, and the other one is in a discrete-time setting. Following the pioneering work, there has been a growing interest in dynamic pricing of perishable products. The area is surveyed in review papers by Bitran and Caldenty (2003), Elmaghraby and Keskinocak(2003), Chiang et al. (2007), Chen and Chen (2015) [1][4]-[6]. In E-commerce times, prices can be easily adjusted over time at little or no cost and most consumers have accepted the multiple-price pattern. As a result, dynamic pricing becomes a more popular pricing strategy in business practice. However, here arises a new problem related to behavioral issues. If the selling season is divided into multiple periods and different prices are set for different periods, the past prices will also determine customers' purchase decisions as the current price. The reason is that when customers arrives, they usually develop reference

*Corresponding author: yanghui@njust.edu.cn prices (related to past prices), which become the benchmark against which current prices are compared. Prices above the reference price appear to be "high" and depress the demand, whereas prices below the reference price are perceived as "low" and stimulate the demand. The impact of the reference price on demand, called the reference effect, appears inevitably with the adoption of dynamic pricing techniques. The purpose of this paper is to investigate the optimal pricing strategies with reference effects in revenue management settings.

Some recent work investigates dynamic pricing strategies in reference effect settings. Popescu and $\mathrm{Wu}$ (2007) examine the dynamic pricing problem of a monopoly in a market with repeated interactions, where consumers' price expectations follow an exponentially smoothed adaptive expectation process [7]. In the following work, Nasiry and Popescu (2011) explore a different memory-based reference price model, based on the peak-end rule [8]. Coulter and Krishnamoorthy (2014) consider how the reference effect impacts the optimal pricing strategies in competitive situations [9]. These studies work with a deterministic demand model and unlimited capacity. Guler et al. (2014) and Guler et al. (2015) deal with a stochastic demand model, however they consider the initial inventory as a decision variable [10][11]. In our 
paper, the initial capacity is fixed and there is no replenishment opportunity. Our paper combines aspects of many of these papers and expands them. In our models, the firm is faced with a classical revenue management problem with the properties of stochastic demand, finite horizon and fixed capacity. The firm applies a discrete-time dynamic pricing policy and there are continuous allowable prices. In particular, we incorporate a memory-based reference price in the demand model to investigate how the reference effect impacts on traditional revenue management decisions.

The remainder of the paper is organized as follows. Section 2 proposes a fixed pricing model. Section 3 extends it to a periodic pricing model with reference effect. Numerical examples and managerial implications are placed in Section 4, followed by concluding remarks.

\section{A FIXED PRICING MODEL}

Consider a product with limited capacity $N$ sold by a monopolistic firm over a finite planning horizon $[0, T]$. The firm can influence demand by varying its price $p$. The arriving demand is stochastic and characterized as a Poisson process with intensity $\lambda(p)$ (see, e.g. Gallego and van Ryzin, 1994). We define $J(p)$ as the number of actual demand at price $p$. Therefore, the probability mass function for the actual number is given by

$$
\operatorname{Pr}\{J(p)=j\}=\frac{(\lambda(p) T)^{j}}{j !} e^{-\lambda(p) T}
$$

From an analytical perspective, we assume there is a one-to-one linear correspondence between prices and demand rates $\lambda(p)=a-b p, a>0, b>0$. The firm is to determine a target price which brings a unique demand rate. There exists a very high price $\bar{p}$ and the firm won't post the price higher than it for some reasons, e.g., demand constraints or government regulations. For simplicity, the salvage value for the product is set to zero. We assume the firm applies a static price policy. His expected revenue function is

$$
r(p)=\sum_{j=0}^{\infty} \min (N, j) p \cdot \operatorname{Pr}\{J(p)=j\}
$$

By replacing the probability mass function by its corresponding formula, equation (1) can be rewritten as follows:

$$
r(p)=\sum_{j=0}^{N} \frac{(\lambda(p) T)^{j}}{j !} e^{-\lambda(p) T} p j+\sum_{j=N+1}^{\infty} \frac{(\lambda(p) T)^{j}}{j !} e^{-\lambda(p) T} p N
$$

The optimal price $p^{*}$ is given by

$$
p^{*}=\underset{p \in[0, \bar{p}]}{\arg \max }\left(p N-p \cdot \sum_{j=0}^{N} \frac{(\lambda(p) T)^{j}}{j !} e^{-\lambda(p) T} \cdot(N-j)\right)
$$

The existence of a unique solution to equation (2) is resolved by the following proposition.

Proposition 1: There exists a unique optimal solution to the static pricing model. The optimal price $p^{*}=\bar{p}$ or satisfies $r^{\prime}\left(p^{*}\right)=0$.

Proof:

We denote $r(p)$ as separate functions associated with price $p, r(p)=p N-f(p) g(p)$

where $f(p)=p e^{-\lambda(p) T}$ and $g(p)=\sum_{j=0}^{N} \frac{(\lambda(p) T)^{j}}{j !}(N-j)$.

We can also denote $g(p)$ as

$$
g(p)=\sum_{j=0}^{N} \frac{(\lambda(p) T)^{j}}{j !}(N-j)=N+\sum_{j=1}^{N} \frac{(\lambda(p) T)^{j}}{j !}(N-j) \text {. }
$$

In this way, we can get the first-order and the second-order derivative of $f(p)$ and $g(p)$

$$
\begin{aligned}
f^{\prime}(p) & =e^{-\lambda(p) T}(1+b p T) \\
f^{\prime \prime}(p) & =e^{-\lambda(p) T}\left(b^{2} T^{2} p+2 b T\right) \\
g^{\prime}(p) & =\sum_{j=1}^{N} \frac{(\lambda(p) T)^{j-1}}{(j-1) !}(N-j)(-b T) \\
& =\sum_{j=0}^{N} \frac{(\lambda(p) T)^{j}}{j !}(N-j-1)(-b T) \\
g^{\prime \prime}(p) & =\sum_{j=0}^{N-2} \frac{(\lambda(p) \cdot T)^{j}}{j !} \cdot(N-j-2)(b T)^{2} .
\end{aligned}
$$

Therefore, the first-order derivative of $r(p)$ can be written as

$$
\begin{aligned}
r^{\prime}(p) & =N-\left(f^{\prime}(p) g(p)+f(p)^{\prime} g(p)\right) \\
& =N-\sum_{j=0}^{N-1} \frac{(\lambda(p) T)^{j}}{j !} e^{-\lambda(p) T} \cdot(N-j+b p T) .
\end{aligned}
$$

And the second-order derivative of $r(p)$ can be written as

$$
r^{\prime \prime}(p)=-\left[f^{\prime \prime}(p) g(p)+f(p) g^{\prime \prime}(p)\right]
$$

Two situations are discussed as we consider the concavity of $r(p)$ :

(1) When $N=1, r^{\prime \prime}(p)=-e^{-\lambda(p) T}\left(b^{2} T^{2} p+2 b T\right) \leq 0$ for all prices in the interval $[0, \bar{p}]$.

(2) When $N \geq 2$,

$r^{\prime \prime}(p)=-e^{-\lambda(p) T} \cdot\left[\left(b^{2} T^{2} p+2 b T\right) \frac{(\lambda(p) \cdot T)^{N-1}}{(N-1) !}+2 b t \sum_{j=0}^{N-2} \frac{(\lambda(p) \cdot T)^{j}}{j !}\right]$ which is negative for all prices in the interval $[0, \bar{p}]$.

Therefore, $r(p)$ is concave for all prices in the interval $[0, \bar{p}]$ which indicates the existence of the unique optimal solution to the static pricing model. The optimal price $p^{*}=\bar{p}$ or satisfies $r^{\prime}\left(p^{*}\right)=0$.

This proposition shows that the firm can find a unique optimal price to maximize his revenue if he 
decides to set a static price for the whole selling horizon. Most of the time, the optimal price is given by the first-order optimality condition, which is described as

$$
N-\sum_{j=0}^{N-1} \frac{\left(\lambda\left(p^{*}\right) T\right)^{j}}{j !} e^{-\lambda\left(p^{*}\right) T} \cdot\left(N-j+b p^{*} T\right)=0
$$

\section{PERIODIC PRICING MODEL CONSIDERING REFERENCE EFFECT}

\subsection{Model description}

In practice we observe that suppliers often check their inventories periodically and revise the prices to influence the selling quantities. As the realized sales information is updated dynamically in the pricing decision process, this policy can better match demand with fixed capacity and improve the firm's revenue. We define $K$ as the number of times the price can be revised during the planning horizon and $\Delta t_{k}$ as the length of the $k \operatorname{th}(k=0,1, \ldots K)$ time interval. The firm needs to decide what price to charge to customers from a bounded interval $[0, \bar{p}]$ in each period based on the realized demand information. We define $p_{k}$ as the price in period $k$. Therefore, the firm charges an initial price $p_{1}$ at the beginning of the selling horizon and collect he information of the realized demand when in the end of this period while the price $p_{2}$ for the second period is determined based on the demand information the firm has collected. The decision process repeats till the final period $K$ as shown in Figure 1.

As the firm manipulates prices, customers develop a price expectation while making purchase decisions. We define $p_{k}^{r}$ as the reference price in period $k$ to which the current prices are compared.

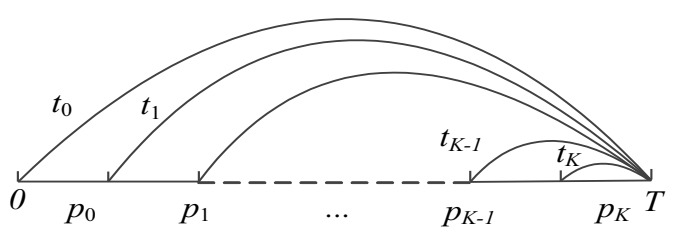

Figure 1. Periodic pricing review policy

\subsection{Demand function and optimal solution}

We model the reference effect in period $k$ as

$R\left(x, p_{k}^{r}\right)=\theta\left(p_{k}^{r}-p_{k}\right)$

Where $\theta>0$ captures the intensity of the reference effect and $x=p_{k}^{r}-p_{k}$, denoting the difference between the reference price and the actual price which causes customers' gains and losses. The exponential smoothing formation mechanism of reference price (Popescu, 2007) is given by $p_{k}^{r}=\mu p_{k-1}^{r}+(1-\mu) p_{k-1}$

where $p_{k-1}^{r}$ and $p_{k-1}$ denote the reference price and the actual price in period $k-1$. We define $\mu \in[0,1)$ as the memory parameter which characterizes the dependence of reference price on history prices. A lower value of $\mu$ represents that customers have less memory of history prices and the extreme situation of $\mu=0$ indicates that the reference price $p_{k}^{r}$ equals to the past price $p_{k-1}$.

We assume a liner correspondence between prices and demand rates when no reference effect is considered, which is given by

$\lambda\left(p_{k}\right)=a-b p_{k}, a>0, b>0$.

We assume that the demand rate with reference effect is an additive function of $\lambda\left(p_{k}\right)$ and $R\left(x, p_{k}^{r}\right)$, which can be written as

$\lambda\left(p_{k}^{r}, p_{k}\right)=a-b p_{k}+R\left(x, p_{k}^{r}\right)$

Prices below the reference price increase customers' demands by generating a loss perception while prices above the reference price decrease customers' demands by generating a gain perception. The latter effect may somehow be negative to the firm. Meanwhile, the fixed pricing policy exerts no reference effect on customers. Therefore, under the reference effect, the periodic pricing review policy could probably reduce the firm's revenue despite its advantage in "precisely pricing". In this section, we construct a periodic pricing model to find out an optimal pricing policy under reference effect and figure out whether a dynamic pricing policy is superior to a fixed pricing policy in spite of the effect of reference prices. We can infer the demand rate in every period using equation (5) and equation (7) classified in two situations:

When $k=1$, there's no past price and no reference effect, which means the reference price in period 1 equals to the current price, namely, $p_{1}^{r}=p_{1}$. Therefore, the demand rate can be written as $\lambda\left(p_{1}, p_{1}^{r}\right)=\alpha-\beta p_{1}$.

When $k \geq 2, \lambda\left(p_{k}, p_{k}^{r}\right)=\alpha-\beta p_{k}+\theta\left(p_{k}^{r}-p_{k}\right)$.

We define $J\left(p_{k}\right)$ as the number of actual demand in period $k$ when the price is $p_{k}$. Therefore, the probability mass function for the actual number in period $k$ is given by

$\operatorname{Pr}\left\{J\left(p_{k}\right)=j\right\}=\frac{\left(\lambda\left(p_{k}, p_{k}^{r}\right) t_{k}\right)^{j}}{j !} e^{-\lambda\left(p_{k}, p_{k}^{r}\right) t_{k}}$

We define the function $r\left(p_{k}\right)$ as the expected revenue from period $k$ till the last period $K$ if the initial inventory of period $k$ is $n_{k}$ and the price is $p_{k}$. 
Therefore,

$r\left(p_{k}\right)=\sum_{j=0}^{n_{k}} \min \left(n_{k}, j\right) p_{k} \cdot \operatorname{Pr}\left\{J\left(p_{k}\right)=j\right\}$

By replacing the probability mass function by its corresponding formula, equation (8) can be rewritten as follows:

$$
\left.r\left(p_{k}\right)=n_{k}-p_{k} \sum_{j=0}^{n_{k}} \frac{\left(\lambda\left(p_{k}, p_{k}^{r}\right) t_{k}\right)^{j}}{j !} e^{-\lambda\left(p_{k}, p_{k}^{r}\right) t_{k}}\left(n_{k}-j\right)\right)
$$

The optimal price $p^{*}$ is given by

$$
p_{k}^{*}=\underset{p \in[0, \bar{p}]}{\arg \max } r\left(p_{k}\right)
$$

The existence of a unique solution to this equation is resolved by the following proposition, which can be proved in a similar way to proposition 1 .

Proposition 2: There exists a unique optimal solution to the periodic pricing model. The optimal price $p_{k}^{*}=\bar{p}$ or satisfies $r^{\prime}\left(p_{k}{ }^{*}\right)=0$.

Most of the time, the optimal price is given by the first-order optimality condition, which is described as

$$
n_{k}-\sum_{j=0}^{n_{k}-1} \frac{\left(\lambda\left(p_{k}^{*}, p_{k}^{r}\right) t_{k}\right)^{j}}{j !} e^{-\lambda\left(p_{k}^{*}, p_{k}^{r}\right) t_{k}} \cdot\left(n_{k}-j+b p_{k}^{*} t_{k}\right)=0(9)
$$

It is quite difficult to obtain its closed-form solution. However, we can get the numerical solution easily by some computing software, such as Matlab.

\section{NUMERICAL EXAMPLES}

Table 1. Values of the parameters

\begin{tabular}{lllllll}
\hline & $N$ & $T$ & $\alpha$ & $\beta$ & $\mu$ & $\Theta$ \\
\hline High demand & 20 & 10 & 10 & 1 & 0.8 & {$[0,0.6,3,5]$} \\
Low demand & 20 & 10 & 2 & 1 & 0.8 & {$[0,0.6,3,5]$} \\
\hline
\end{tabular}

In this section, we analyze the numerical results of the fixed pricing model proposed in section 2 and the period pricing policy proposed in section 3 . We consider two situations: the high demand situation in which the firm sells out all products and the low demand situation in which there are unsold products at the end the selling horizion. We set four different values of $\theta$ to represent different levels of reference effect. The parameters are listed in Table 1.

\subsection{High demand situation}

Table 2 lists the optimal prices and expected revenues with different levels of reference effect and different review times when the demand is high. Figure 2 and Figure 3 describe the optimal price paths and revenue curves. The situation $K=0$ represents the fixed pricing policy and $\theta=0$ denotes the condition without reference effect.
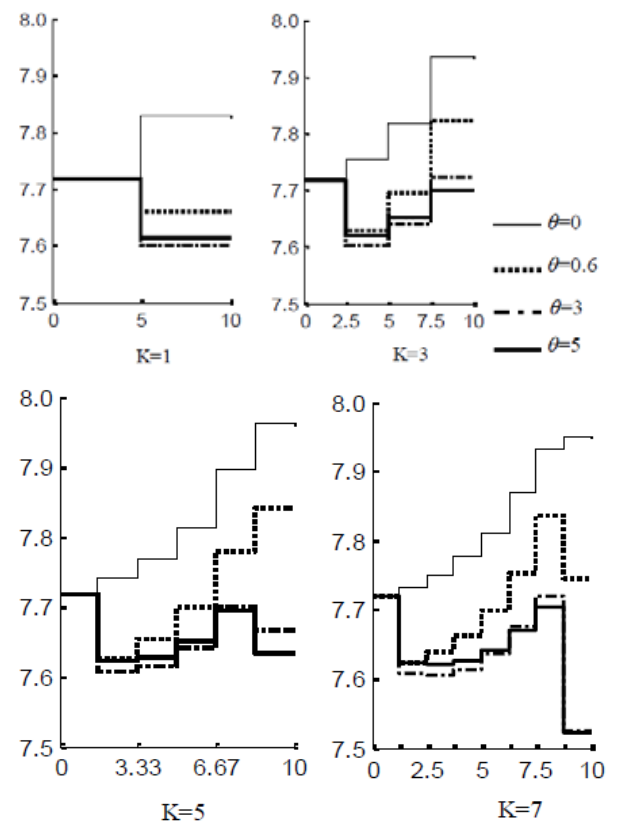

Figure 2. The optimal price paths in high demand situation

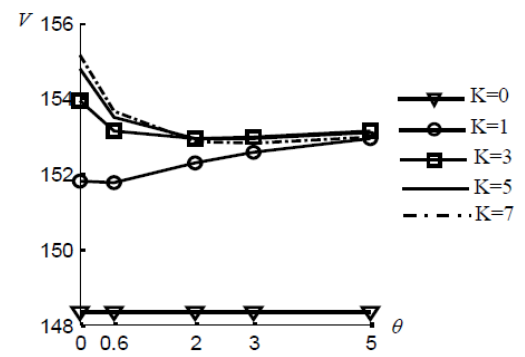

Figure 3. The expected revenues in high demand situation

The numerical results show that: Firstly, firms should adopt markup strategy in the high demand situation when no reference effect is considered. When considering reference effect, firms should adopt skimming strategy to anchor a high initial price and the markdown strategy is taken in the subsequent periods (see Table 2 and Figure 2). Secondly, compared to the situation without reference effect, firms under the situations with reference effect sell more products but gain less revenue. Thirdly, the periodic price review policy gains more revenue than the fixed pricing policy whether reference effect is considered or not (see Figure 3). Fourthly, the revenue of the firm increases as the reference effect gets stronger in the situation that firms update prices in a low frequency, say, $K=1$. When firms update prices in a higher frequency the revenue decreases with the intensity of the reference effect.

The numerical results provide several management implications:

a. Markup strategy VS. Skimming strategy

The markup strategy is effective when firms ob- 
ICITCE 2015

Table 2. The optimal prices and expected revenues in high demand situation

\begin{tabular}{|c|c|c|c|c|c|c|c|c|c|c|c|}
\hline \multirow{2}{*}{$\begin{array}{l}\mathrm{K} \\
0\end{array}$} & \multirow{2}{*}{$\frac{\theta}{1}$} & \multicolumn{8}{|c|}{$p_{k}^{*}$} & \multirow{2}{*}{$\begin{array}{l}\text { Expected demand } \\
19.22\end{array}$} & \multirow{2}{*}{$\begin{array}{l}\text { Expected revenue } \\
148.38\end{array}$} \\
\hline & & 7.72 & & & & & & & & & \\
\hline \multirow{4}{*}{11} & 0 & 7.72 & 7.83 & & & & & & & 19.55 & 151.79 \\
\hline & 0.6 & 7.72 & 7.66 & & & & & & & 19.72 & 151.74 \\
\hline & 3 & 7.72 & 7.60 & & & & & & & 19.90 & 152.56 \\
\hline & 5 & 7.72 & 7.61 & & & & & & & 19.93 & 152.94 \\
\hline \multirow{4}{*}{3} & 0 & 7.72 & 7.75 & 7.82 & 7.93 & & & & & 19.76 & 153.93 \\
\hline & 0.6 & 7.72 & 7.63 & 7.70 & 7.82 & & & & & 19.89 & 153.11 \\
\hline & 3 & 7.72 & 7.60 & 7.64 & 7.72 & & & & & 19.97 & 152.95 \\
\hline & 5 & 7.72 & 7.62 & 7.65 & 7.70 & & & & & 19.99 & 153.14 \\
\hline \multirow{4}{*}{5} & 0 & 7.72 & 7.74 & 7.77 & 7.81 & 7.90 & 7.96 & & & 19.85 & 154.78 \\
\hline & 0.6 & 7.72 & 7.62 & 7.65 & 7.70 & 7.78 & 7.84 & & & 19.94 & 153.49 \\
\hline & 3 & 7.72 & 7.61 & 7.61 & 7.64 & 7.70 & 7.66 & & & 19.99 & 152.92 \\
\hline & 5 & 7.72 & 7.62 & 7.63 & 7.65 & 7.69 & 7.63 & & & 20.00 & 153.08 \\
\hline \multirow{4}{*}{7} & 0 & 7.72 & 7.73 & 7.75 & 7.78 & 7.81 & 7.87 & 7.93 & 7.95 & 19.89 & 155.15 \\
\hline & 0.6 & 7.72 & 7.62 & 7.64 & 7.66 & 7.70 & 7.75 & 7.84 & 7.75 & 19.96 & 153.64 \\
\hline & 3 & 7.72 & 7.61 & 7.61 & 7.61 & 7.63 & 7.68 & 7.72 & 7.52 & 19.99 & 152.82 \\
\hline & 5 & 7.72 & 7.62 & 7.62 & 7.63 & 7.64 & 7.67 & 7.70 & 7.52 & 20.00 & 152.97 \\
\hline
\end{tabular}

Table 3. The optimal prices and expected revenues in low demand situation

\begin{tabular}{|c|c|c|c|c|c|c|c|c|c|c|c|}
\hline \multirow{2}{*}{$\begin{array}{l}\mathrm{K} \\
0\end{array}$} & \multirow{2}{*}{$\frac{\theta}{1}$} & \multicolumn{8}{|c|}{$p_{k}^{*}$} & \multirow{2}{*}{$\begin{array}{l}\text { Expected demand } \\
9.98\end{array}$} & \multirow{2}{*}{$\begin{array}{l}\text { Expected revenue } \\
9.98\end{array}$} \\
\hline & & 1.00 & & & & & & & & & \\
\hline \multirow{4}{*}{11} & 0 & 1.00 & 1.00 & & & & & & & 9.99 & 10.00 \\
\hline & 0.6 & 1.00 & 0.81 & & & & & & & 11.48 & 10.28 \\
\hline & 3 & 1.00 & 0.68 & & & & & & & 16.14 & 12.57 \\
\hline & 5 & 1.00 & 0.71 & & & & & & & 17.78 & 14.08 \\
\hline \multirow{4}{*}{3} & 0 & 1.00 & 1.00 & 1.00 & 1.00 & & & & & 9.99 & 10.00 \\
\hline & 0.6 & 1.00 & 0.82 & 0.81 & 0.80 & & & & & 12.14 & 10.30 \\
\hline & 3 & 1.00 & 0.74 & 0.70 & 0.65 & & & & & 17.72 & 13.09 \\
\hline & 5 & 1.00 & 0.78 & 0.74 & 0.71 & & & & & 19.01 & 14.81 \\
\hline \multirow{4}{*}{5} & 0 & 1.00 & 1.00 & 1.00 & 1.00 & 1.00 & 1.00 & & & 10.00 & 10.00 \\
\hline & 0.6 & 1.00 & 0.82 & 0.81 & 0.80 & 0.80 & 0.79 & & & 12.30 & 10.22 \\
\hline & 3 & 1.00 & 0.75 & 0.72 & 0.69 & 0.65 & 0.61 & & & 18.02 & 12.87 \\
\hline & 5 & 1.00 & 0.79 & 0.76 & 0.73 & 0.71 & 0.68 & & & 19.26 & 14.62 \\
\hline \multirow{4}{*}{7} & 0 & 1.00 & 1.00 & 1.00 & 1.00 & 1.00 & 1.00 & 1.00 & 1.00 & 10.00 & 10.00 \\
\hline & 0.6 & 1.00 & 0.82 & 0.81 & 0.80 & 0.80 & 0.79 & 0.79 & 0.78 & 12.36 & 10.14 \\
\hline & 3 & 1.00 & 0.76 & 0.73 & 0.70 & 0.67 & 0.64 & 0.61 & 0.58 & 18.07 & 12.51 \\
\hline & 5 & 1.00 & 0.80 & 0.77 & 0.74 & 0.71 & 0.69 & 0.67 & 0.64 & 19.35 & 14.29 \\
\hline
\end{tabular}

serve a booming market as the marginal revenue of each product which will be maximized. And the inhibiting effects on demands attributed to low reference prices generated by this strategy in the situation with reference effect can be weaken by adopting skimming strategy in which a high initial price is posted to establish a high reference price.

b. Period pricing review policy VS. Fixed pricing policy

In spite of the weakening effect on revenue caused by reference prices under markup strategy, the period pricing review policy is still superior to the fixed pricing policy as the skimming strategy is adopted.

c. The optimal updating frequency

The reference dependence of customers could harm the revenue of the firm. But the firm can maximize his revenue by setting the optimal number of times the price should be revised according to the level of reference effect.

\subsection{Low demand situation}

Table 3 lists the optimal prices and expected revenues in situations with low demand. Figure 4 and Figure 5 illustrate the optimal price paths and revenue curves with different levels of reference effect and different review times. $K=0$ represents the fixed pricing policy and $\theta=0$ denotes the condition without reference effect.

Table 3 Figure 4 and Figure 5 show the following results: Firstly, the optimal prices approach to a constant steady-state path in the situation without reference dependence. When the reference effect is considered firms should adopt markdown strategy in 
which the initial price is much higher than the prices in the subsequent periods. Secondly, compared to the situation where customers behavior with no reference effect, firms under the reference effect sell more products and gain more revenue. Thirdly, the period pricing review policy has no obvious advantage over the fixed pricing policy in the condition with no reference effect. However, influenced by the reference dependence of the customers, the former gains much more revenue than the latter one. Fourthly, the revenue of the firm increases as the reference effect gets stronger.
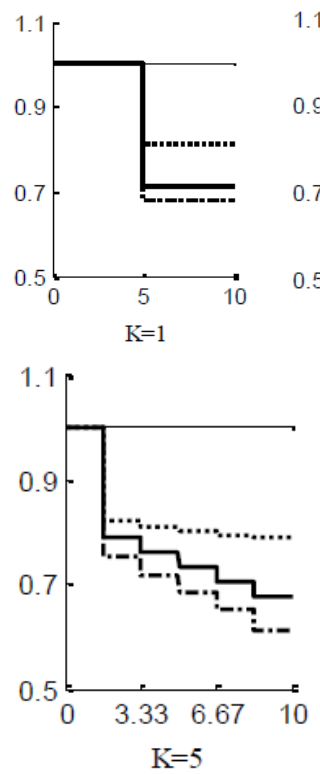

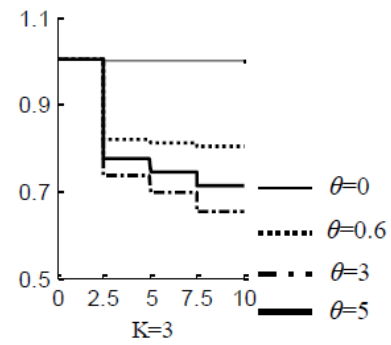

1.1

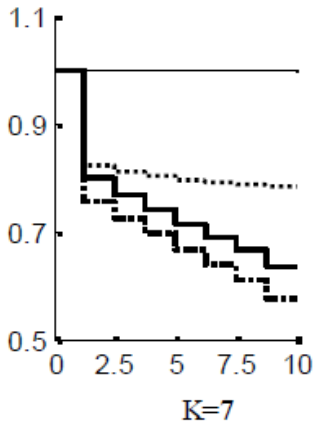

Figure 4. The optimal price paths in low demand situation

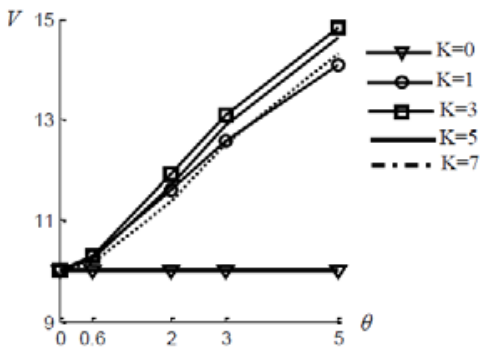

Figure 5. The expected revenues in low demand situation

The numerical results provide several management implications:

a. Constant price VS. Price dispersion

Markets with low demands are advisable for variable constant prices but the reference effect would aggravate price dispersion since higher price sensitivity is formed owing to variable reference prices.

b. Period pricing review policy VS. Fixed pricing policy

Sluggish markets lower the firm's intention of significant price adjustments because of the low price sensitivity of customers. Therefore, the fixed pricing policy can be effective in the situation with no refer- ence effect. However, a markdown strategy should be adopted when customers behave under the effect of reference dependence since it generates high reference prices which stimulate customers' sensitivity to prices.

c. The optimal updating frequency

The reference dependence of customers increases the firm's revenue. There exists an optimal frequency of price updating under each level of reference effect.

\section{CONCLUSIONS}

This paper investigates the optimal pricing strategies with reference effects in revenue management settings We firstly propose a static pricing model and prove the existence and uniqueness of the solution. In our models, the firm is faced with a classical revenue management problem with the properties of stochastic demand, finite horizon and fixed capacity. We then extend the fixed pricing model to a periodic pricing model and incorporate a memory-based reference price in the demand function to investigate how the reference effect impacts on traditional revenue management decisions. We present numerical examples in both low demand situations and high demand situations for different levels of reference effects and different updating frequencies. The results show that the dynamic pricing strategies are superior to a static one even when reference effects are taken into consideration. We also provide some managerial insights including pricing directions, pricing dispersion and the optimal updating frequency for both demand situations.

However, we only consider a forward updating policy in our periodic pricing model. The reverse updating policy and the dynamic programming model will be taken into consideration in further research.

\section{ACKNOWLEDGEMENT}

This paper is sponsored by research grants from the National Natural Science Foundation of China (Grant No. 71102073, 71571102)

\section{REFERENCES}

[1] W.C. Chiang, C.H. Jason \& X.J. Xu. 2007. An overview of research on revenue management: Current issues and future research. International Journal of Revenue Management, 1(1): 97-128.

[2] G. Gallego \& G.V. Ryzin. 1994. Optimal dynamic pricing of inventories control. Management Science, 40(8): 999-1020.

[3] G. Bitran \& S.V. Mondschein. 1997. Periodic pricing of seasonal products in retailing. Management Science, 43: 64-79.

[4] G. Bitran \& R. Caldentey. 2003. An overview of pricing models for revenue management. Manufacturing \& Service Operations Management, 5: 203-229. 
[5] W. Elmaghraby \& P. Keskinocak. 2003. Dynamic pricing in the presence of inventory considerations: Research overview, current practices, and future directions. Management Science, 49: 1287-1309.

[6] M. Chen \& Z. L. Chen. 2015. Recent developments in dynamic pricing research: multiple products, competition, and limited demand information. Production and Operations Management, 24(5): 704-731.

[7] J. Popescu \& Y. Wu. 2007. Dynamic pricing strategies with reference effect. Operations Research, 55: 413-429.

[8] J. Nasiry \& I. Popescu. 2011. Dynamic pricing with loss-averse consumers and peak-end anchoring. Operations Research, 59: 1361-1368.
[9] B. Coultera \& S. Krishnamoorthy. 2014. Pricing strategies with reference effects in competitive industries. International Transactions in Operational Research, 21: 263-274.

[10] M.G. Güler, T. Bilgiç \& R. Güllü. 2014. Joint inventory and pricing decisions with reference effects. IIE Transactions, 46: 330-343.

[11] M.G. Güler, T. Bilgiç \& R. Güllü. 2015. Joint pricing and inventory control for additive demand models with reference effects. Annals of Operations Research, 226: 255-276. 